\title{
Forecasting Model for Crude Oil Prices Based on Artificial Neural Networks
}

\author{
\#Imad Haidar ${ }^{1}$, Siddhivinayak Kulkarni ${ }^{1}$, and Heping Pan $^{2}$ \\ ${ }^{I}$ School of Information Technology and Mathematical sciences, University of Ballarat \\ Ballarat VIC. 3353 Australia \\ PO Box 663 \\ Email: ihaidar@students.ballarat.edu.au,s.kulkarni@ballarat.edu.au \\ ${ }^{2}$ University of Electronic Science and Technology of China, \\ Chengdu 610054, China \\ Email:panhp@swingtum.com
}

\begin{abstract}
This paper presents short-term forecasting model for crude oil prices based on three layer feedforward neural network. Careful attention was paid on finding the optimal network structure. Moreover, a number of features were tested as an inputs such as crude oil futures prices, dollar index, gold spot price, heating oil spot price and $S \& P 500$ index. The results show that with adequate network design and appropriate selection of the training inputs, feedforward networks are capable of forecasting noisy time series with high accuracy.
\end{abstract}

\section{INTRODUCTION}

Crude oil is a key commodity for the economy. However, a major characteristic of crude oil markets is significant price fluctuations. This volatility of oil prices could be attributed to three main factors:

- Supply/demand imbalance, possibly caused by:

$>$ Economical growth.

$>$ Oil producing countries behaviors.

- Exogenous events (war, extreme weather...etc).

- Endogenous factors (speculation in the markets).

The effect of increasing price and its daily fluctuations affect not only the economies and financial markets but extend to reach individuals. This is because an increase in oil prices has a direct effect on petrol prices, in addition, it also affects the prices of other goods and services. Therefore, forecasting crude oil is very important task to reduce the impact of price fluctuations, and help investors, hedgers, and individual to make informed decisions when dealing with energy markets. However, for the above reasons forecasting crude oil is not an easy task.

In this paper, we present an ANN model for crude oil price prediction for the short-term. In addition we test whether crude oil futures prices ${ }^{1}$ contain newer information about spot price direction on the short-term.

This paper proceeds as follows, Section 2 represents short literature review, Section 3 presents data description, and preprocessing along with our methodology. Section 4 details the results and discussion, and finally the paper is concluded in Section 5 .

\section{LITERATURE REVIEW}

The importance of crude oil to the economy is reflected by the number of studies in this area. There are large and rich literature related to every aspect of crude oil. However, until recently the majority of studies were based on either analytical or linear models. While analytical models has failed completely in producing good forecast for crude oil $[2,3]$ linear models such as Box-Jenkins model (ARMA) have provided better results [3]. Nevertheless, it is been believed that crude oil prices are nonlinear time series, hence nonlinear models such as ANN should be superior to other linear models .

Moshiri and Foroutan [3] compared linear and nonlinear models for forecasting crude oil futures prices. The authors compared ARMA and GARCH, to ANN, and found that ANN is superior and produced a statistically significant forecast.

Xie, et al [4] proposed SVM model for monthly crude oil prices prediction, the authors claimed that SVM outperformed feedforward network with backprorogation

\footnotetext{
${ }^{1}$ Futures contracts are defined as: 'A firm commitment to make or accept delivery of specified quantity and quality of commodity during a specific month in the future at price agreed upon at the time the commitment is made' [1] p. 6.
} 
(BPNN) and ARIMA for out-of-sample. However, their results were not consistent, as BPNN outperformed SVM for two of the four sub periods tested. Nonetheless, both BPNN and SVM outperformed ARIMA for all four periods.

Liu et al [5] present hybrid model based on fuzzy neural model to forecast Brent crude oil prices. Three forecasting models were used viz. Redial Based Network, Markov chain based semi parametric model and wavelet analysis based forecasting model. The output of the three methods was used as an input to fuzzy neural network, while the target was the actual Brent crude oil price. The authors concluded that the nonlinear combination outperformed any single model tested. However, the authors based this conclusion on one performance metric only, the root mean square error.

In a related study $\mathrm{Yu}$ et al [6] proposed a decomposing method for time series before training with ANN. Empirical Mode Decomposition (EMD) was applied on daily crude oil spot price which decomposes the original time series into a number intrinsic mode components and a residual. Only six of these signals were selected as input to feedforward network along with the original series. The authors concluded that this method of decomposing outperformed using the original series solely.

Wang et al [7] presented a hybrid methodology to forecast crude oil monthly prices. The model consists of combination of three separate components, Web mining from which the authors extract rule based system, in addition ANN, and ARIMA models. These three components work disjointedly, and then intergraded together to get the final results. They claimed that nonlinear integration of these three models has outperformed any single one. However, in our opinion there are several issues in this system. For example, the rule base system of the text mining model ${ }^{2}$ depends on the knowledge base which developed by human experts. This process is not only controversial, but also unreliable, because experts' opinions vary on the same problem. Moreover, neither the rules nor the knowledge base was made available to the public.

Most of the studies above were based on monthly prices. On one hand using monthly prices reduces the noise in the data, however, on the other hand, it also limits the data size significantly, and force to use old data form the 70's which could be irrelevant to the current economical situations. Beside, limited data also will affect the conclusion as the testing set is barely statistically significant. Most of the studies were concentrated on developing new techniques, but little attention was paid in testing different inputs. The output of any model (linear or nonlinear) is affected significantly by how much information the input contains.

Of course using the historical price of the time series itself is valid (as most of the studies have done) however, it is by no

\footnotetext{
${ }^{2}$ For a survey of text mining for financial prediction see [8]
}

means enough. In this context, our model is based on multivariate inputs to forecast the short term-direction of crude oil prices aiming to cover a gap in nonlinear forecasting of crude oil.

\section{METHODOLOGY}

Our objective is to build a model for short-term forecast of the crude oil price direction. Backpropgation feedforward networks BFFNN was selected as nonlinear mapping model as it does not make any assumption about the problem; rather it try to find the appropriate function from the data itself [9]. Furthermore, ANN is considered as general function approximator method [10]. Nevertheless, ANN has some limitations such as, the tendency to over fit the data. Therefore, in order to build effective model careful attention was paid on finding the optimal network architecture, as well as selecting informative inputs for training the network. However, in order to detriment whether ANN class models are suitable for the crude oil data or not, the structure of the data need to be confirmed.

\section{A. Testing for normality and nonlinearity}

We tested the distribution of the crude oil spot data, and the existence of nonlinearity. If the data are not normally distributed and more importantly not linear then modelling with linear model such as ARMA is not appropriate. Table 1 presents the results of Jarque-Bera (J-B) test. Under the null hypothesis the data is normally distributed.

TABLE 1 J-B TEST FOR CRUDE OIL SPOT DATA
\begin{tabular}{|c|c|c|c|}
\hline Series & P value & J-B stat & C value \\
\hline Spot return & $<0.01$ & 2195.925 & 5.9701 \\
\hline
\end{tabular}

Based on the results in Table 1, we can reject the null hypotheses of normal distribution. The Brock, Dechert and Scheinkman test (BDS) was conducted for nonlinearity ${ }^{3}$. BDS test is by far the most used test for nonlinearity in the data. We apply the test on crude oil spot relative change. Linear regression was applied on the original return to remove the linearity from the data and the BDS test was applied on the residuals. The test was used for the embedded dimension of $\mathrm{N}=2$ to 20 and two different values of $e$ were selected 1 , and 1.5 of the stranded deviation with $e=1$ equals to the stranded deviation of the data. For $e=1$ the data reject the null hypothesis of independently identically distributed which indicates the existence of nonlinear structure in the data. The same conclusion can be obtained for $e=1.5$ and for all value of $\mathrm{N}$ tested. Moreover, the results show that the evidence of nonlinearity in the data has increased with higher dimensions as the $\mathrm{W}_{(\mathrm{N}) \mathrm{e}}$ increased with larger values of $\mathrm{N}$ and $e$. In light of these results nonlinear models such as ANN can be used for modeling with this data.

\footnotetext{
3 The MATLAB code for BDS test used in this section was written by Ludwig Kanzler 1998 and retrieved from: http://ww61.tiki.ne.jp/ kanzler/\#L.\%20Kanzler:\%20Software
} 
TABLE 2 BDS TEST FOR CUDE OIL SPOT DATA LINEARLY FILERTRED

\begin{tabular}{|c|c|c|c|c|}
\hline $\mathrm{N}$ & $\mathrm{e}$ & $\mathrm{W}_{(\mathrm{N}) \mathrm{e}}$ & $\mathrm{e}$ & $\mathrm{W}_{(\mathrm{N}) \mathrm{e}}$ \\
\hline 2 & 1.5 & 4.7963 & 1 & 3.2218 \\
\hline 3 & 1.5 & 6.2515 & 1 & 4.1548 \\
\hline 4 & 1.5 & 6.9357 & 1 & 4.8923 \\
\hline 5 & 1.5 & 7.2325 & 1 & 5.1992 \\
\hline 6 & 1.5 & 7.7066 & 1 & 5.8902 \\
\hline 7 & 1.5 & 8.2214 & 1 & 6.6529 \\
\hline 8 & 1.5 & 8.5258 & 1 & 7.2729 \\
\hline 9 & 1.5 & 8.8533 & 1 & 7.9657 \\
\hline 10 & 1.5 & 9.0754 & 1 & 8.7041 \\
\hline 11 & 1.5 & 9.2945 & 1 & 9.556 \\
\hline 12 & 1.5 & 9.4367 & 1 & 10.296 \\
\hline 13 & 1.5 & 9.6892 & 1 & 11.357 \\
\hline 14 & 1.5 & 9.9989 & 1 & 12.492 \\
\hline 15 & 1.5 & 10.382 & 1 & 13.799 \\
\hline 16 & 1.5 & 10.802 & 1 & 15.478 \\
\hline 17 & 1.5 & 11.180 & 1 & 17.290 \\
\hline 18 & 1.5 & 11.632 & 1 & 19.331 \\
\hline 19 & 1.5 & 12.160 & 1 & 21.659 \\
\hline 20 & 1.5 & 12.757 & 1 & 24.526 \\
\hline
\end{tabular}

The critical values are $1.645,1.96$ and 2.575 for the $10 \%, 5 \%$, and $1 \%$ significant level consequently.

\section{B. Features Selection:}

The choice of inputs-outputs is crucial for BFFNN training process; if the inputs contain no information about the target market then it will disadvantage the learning process and could generate poor generalization. The outstanding problem for crude oil short-term forecast is that fundamental variables such as supply, demand inventory, are not available on daily frequency. Thus, based on our understanding of the energy market dynamics, two groups of features were considered as inputs. Group A consists of crude oil futures prices, and group B inter-market data. The fundamental idea of using futures prices to forecast spot price is based on the assumption that it is much more convenient for market participants to react to new information by taking a position in futures rather than spot prices [11]. This is because futures contracts require low transaction cost, easy to sell and low credit risk [11].

Figure 1 shows a plot of spot price and futures contracts 1, 2, 3 and 4 months to maturity ${ }^{4}$. Although the literature body on using futures prices to predict spot price is substantial [1115], however, there is no consistent evidence of reliability of futures as spot predictors. Further some economists believe that futures prices should not contain any new information otherwise it will violate the market efficiency condition and create an arbitrage opportunity [16]. While others believe that futures prices will not continue to have more information about spot price longer than half-hour, and therefore modeling with daily prices is not sufficient [17]. Therefore, our approach is to consider other features as inputs and compare the performance.

\footnotetext{
${ }^{4}$ For illustration purposes only the last 70 data point were plotted.
}

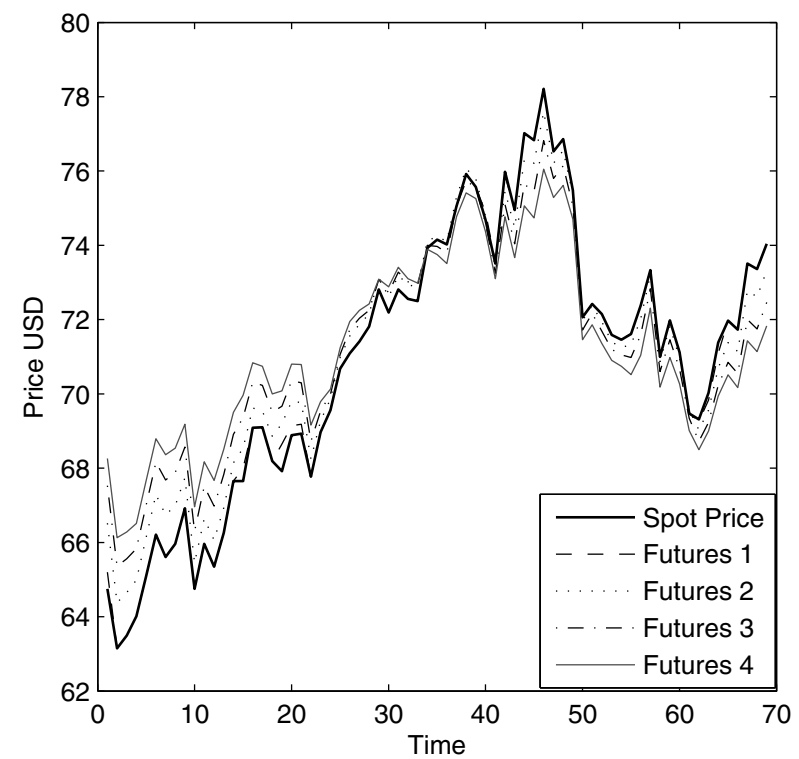

Fig. 1: A plot of the last 70 data points of spot price and 4 futures contracts

Group B of features consist of S\&P 500 to represent the market performance, gold spot price, as important commodity that less volatile than crude oil and could reflect the real trend in the commodity market rather than the noise. In addition, the dollar index, to show the strength of the USD compared to a basket of international currencies ${ }^{5}$. And finally, the heating oil \#2 spot price, as indication of seasonality in the energy market.

The overall strategy is to create a benchmark based on current and lagged value of crude oil spot price solely, and then compare it to the performance of each group when added to the benchmark as an input.

Energy data used in this study consists of West Texas Intermediate (WTI) light sweet crude oil spot price and futures contracts $1,2,3$ and 4 months to maturity traded at NYMEX, In addition to US heating oil \#2 spot price. These data sets were retrieved from US Department of energy: Energy Information Administration: http://www.eia.doe.gov/. On the other hand, gold spot price and US dollar index purchased from Normans Historical Data from: http://www.normanshistoricaldata.com. Finally the S\&P500 data was downloaded from yahoo finance from http://finance.yahoo.com/. All datasets represent daily closing price from 1996 to Aug 2007, it includes 2912 data points for each time series. The data was divided chronologically into training and testing sets, we use $90 \%$ of the data for training and $10 \%$ for out-of-sample testing (approximately one financial year). Further, as early stopping was used to control the training process, the training data was divided into $60 \%$ training and $20 \%$ for testing and $20 \%$ for validation.

5 These currencies are: Euro, Japanese yen, Pound sterling, Canadian dollar, Swedish krona, and Swiss franc. 


\section{ANN Design Considerations:}

In the broadest sense, there are three main requirements for any successful ANN model [9] p.15:

- In-sample accuracy.

- The ability of the model to perform with new data.

- Stability, consistency of the network output.

To insure the above points are successfully met, a large number of considerations need to be taken into account.

Our tests included data pre-processing techniques the number of layers, the choice of activation function, learning rate, training time, optimization algorithms (first, and secondorder) and the number of hidden neurons. After several combinations of experiments, the architecture was finalized for all the main experiments. The most critical issue when dealing with neural networks is to determine the number of hidden neurons as too many hidden neurons will result in over fitting and too few could result in under fitting. The goal is to use the least amount of neurons which generate the best results for out-of-sample [18]. There are no formal rules to solve this dilemma. Heuristics algorithms and evolutionary computing methods are often used. Each of these methods has its advantages and disadvantages. A simple approach due to [19] is based on starting with very small numbers of neurons and training and testing the networks to a fixed number of iterations. The hidden neurons increased gradually until the optimal number of neurons is found. This method was also implemented by [20] to successfully develop an ANN trading system for the Australian stock market. A similar approach was used in this paper; every time a new input (or lagged value of the same variable) was added, we started with 1 hidden neuron and added one each time up to 10. Moreover, each network for each number of neurons was tested three times with different sets of weights to ensure stability. Then the results from the three trials were averaged to get a representative conclusion about each models performance.

\section{Performance Measures:}

The ultimate goal of this study is to forecast the direction of the price, since it is very difficult, if not impossible, to correctly predict the magnitude of the price for financial data. Besides, our aim is not profitability rather risk management, hence predicting the direction is sufficient to fulfill this goal. The success ratio for direction prediction (or the hit rate) was considered [21].

$$
h=\frac{1}{n} \sum_{n=1}^{n} z
$$

$z=1$ if $x_{t+1} \cdot o_{t+1}>0$, and 0 otherwise.

where: $n$ is the sample size $x_{t+1}, o_{t+1}$ are the value of the target and the output at time $t+1$ consecutively.
The RMSE is by far the most used metric for ANN performance regardless of the network goal. Furthermore, the correlation coefficient $\mathrm{R}$ and $\mathrm{R}^{2}$ was also used; as a measure of the linear correlation between the forecasted value and the actual one [9]. Mean squared error, means absolute error, and sum squared error were also calculated. Finally, the information coefficient given by equation 2 was used [9].

$$
I c=\frac{\sqrt{\sum_{t=1}^{n}\left(y_{t}-x_{t}\right)^{2}}}{\sqrt{\sum_{t=1}^{n}\left(x_{t}-x_{t-1}\right)^{2}}}
$$

where: $y$ is the predicted value, and $x$ is the actual value. This ratio provides an indication of the prediction compared to the trivial predictor based on the random walk, whereas $I c \geq 1$ indicates poor prediction, and $I c>1$ means the prediction is better prediction than the random walk [9].

\section{RESUltS AND ANALYSIS}

\section{A. The Benchmark}

For the benchmark several data preprocessing was considered, namely, first order difference eq 3 and second order difference eq 4 and combination of both as an input and output [22, 23].

$$
\begin{gathered}
y_{t}=\left(\frac{x_{t}-x_{t-n}}{x_{t-n}}\right) \\
y_{t}=\left(\frac{x_{t}-2 x_{t-n}+x_{t-2 n}}{x_{t-n}}\right)
\end{gathered}
$$

Starting from one lag up to 20 lagged value of the spot price was tested. The results obtained from input transformed by eq 3 were very poor the hit rate was around $53 \%$ for out-ofsample. While the results generated by eq 4 was much better around $65 \%$ (eq 4 contains 2 steps differencing) the combination of eq 3 and 4 as input with eq 4 alone as output seems to produce much better results. Table 2 shows the results of the best performance.

TABLE 2: SUMMARY OF PERFORMANCE MAESERS OF THE

\begin{tabular}{|c|c|c|c|c|c|c|}
\hline Metrics & Hit rate & RMSE & $\mathrm{R}^{2}$ & MSE & MAE & SSE \\
\hline In sample & 74.93 & 0.0231 & 0.587 & 0.000 & 0.017 & 1.325 \\
\hline $\begin{array}{c}\text { Out of } \\
\text { sample }\end{array}$ & 76 & 0.0192 & 0.500 & 0.000 & 0.015 & 0.083 \\
\hline
\end{tabular}

Furthermore, in an attempt to improve the results and reduce the noise in the oil data, 3 days moving average was applied on the raw data then the data was transformed into firs order difference. Once gain the performance was tested with respect to the number of lags (1-20 lags). The network structure for the benchmark consisted of three layers 
feedforward with 6 hidden neurons and 0.01 learning rate. The network was trained with Levenberg-Marquardt algorithm.

TABLE 3: SUMMARY OF PERFORMANCE MAESERS OF THE BENCHMARK

\begin{tabular}{|c|r|c|c|c|c|c|}
\hline Metrics & Hit rate & \multicolumn{1}{c|}{ RMSE } & $\mathrm{R}^{2}$ & MSE & MAE & SSE \\
\hline In sample & 79.45 & 0.0083 & 0.670 & 0.000 & 0.006 & 0.148 \\
\hline $\begin{array}{c}\text { Out of } \\
\text { sample }\end{array}$ & 79.79 & 0.0068 & 0.576 & 0.000 & 0.005 & 0.011 \\
\hline
\end{tabular}

Table 3 shows the results obtained by the moving average transformation for 13 lags. It is evident that moving average transformation has improved the results for (in) and out-ofsample. For the benchmark the $I c$ is 0.58 for in-sample and 0.69 for out-of-sample which means the network is outperforming the trivial predictor.

\section{B. Inputs Group A}

TABLE 4: OUT-OF-SAMPLE PERFORMANCE FOR INPUTS GROUP A

\begin{tabular}{|l|l|l|l|l|l|}
\hline & Fut1 & Fut2 & Fut3 & Fut4 & Fut all \\
\hline Hit rate & 80.44 & 80 & 79.55 & 79.77 & 78.155 \\
\hline RMSE & 0.0059 & 0.006 & 0.0063 & 0.0063 & 0.007 \\
\hline $\mathbf{R}^{2}$ & 0.6806 & 0.6702 & 0.6383 & 0.6346 & 0.5459 \\
\hline IC & 0.6358 & 0.6285 & 0.653 & 0.6539 & 0.7384 \\
\hline MSE & 0.000 & 0.000 & 0.000 & 0.000 & 0.7019 \\
\hline MAE & 0.0046 & 0.0047 & 0.0049 & 0.0049 & 0.000 \\
\hline SSE & 0.0096 & 0.0098 & 0.0107 & 0.0108 & 0.0055 \\
\hline
\end{tabular}

Adding futures to the benchmark (1 lag of futures to 13 lag of transformed spot) did not outperform the benchmark in term of hit rate for in-sample. While for out-of-sample networks contain futures 1 and networks contain futures 2 has outperformed the benchmark (Table 4). Furthermore, there is no significant improvement for RMSE for in-sample, however, it did improve for out-of-sample for each of the futures compared to the benchmark, and futures 1 preformed the best. The $\mathrm{R}^{2}$ was noticeable better for out-of-sample futures contract 1 compared to the benchmark indicating better fit, while for in-sample was less than the benchmark. The information coefficient ratio Ic did not change for insample for all of the contracts, however, it was improved for out-of-sample for all futures contracts especially contract 1 and 2. Overall the performance was improved for out-ofsample and did not change for in-sample.

Finally, adding all the contracts $1,2,3$, and 4 together to the benchmark has disadvantaged the model. It is safe to conclude that futures contracts 1 and 2 months to maturity have improved the out-of-sample prediction, however, this improvement is not significant enough to make concrete conclusion.

\section{Inputs Group B}

As can be seen from Table 5 none of the inputs in group B has outperformed the benchmark the benchmark. In the mater of fact none of the inputs has matched the benchmark.

TABLE 5: OUT-OF-SAMPLE PERFORMANCE FOR INPUTS GROUP B

\begin{tabular}{|l|l|l|l|l|l|}
\hline & S\&P 500 & $\begin{array}{l}\text { Dollar } \\
\text { Index }\end{array}$ & Gold & $\begin{array}{l}\text { Heating } \\
\text { oil }\end{array}$ & All \\
\hline Hit rate & 78.22 & 78 & 77.48 & 79.11 & 77.25 \\
\hline RMSE & 0.0069 & 0.0068 & 0.0069 & 0.0069 & 0.0069 \\
\hline R $^{2}$ & 0.5631 & 0.5749 & 0.573 & 0.5752 & 0.5663 \\
\hline IC & 0.7953 & 0.784 & 0.7862 & 0.784 & 0.7922 \\
\hline MSE & 0.000 & 0.000 & 0.000 & 0.000 & 0.000 \\
\hline MAE & 0.00538 & 0.0054 & 0.0054 & 0.0054 & 0.0054 \\
\hline SSE & 0.0130 & 0.0126 & 0.0126 & 0.0126 & 0.0128 \\
\hline
\end{tabular}

\section{Multi steps forecast}

Since moving average transformation is used, then it is important to test the model ability to forecast for the median point of the moving average. For example if the data transformed into 10 days moving average then it is important to test the model ability to forecast 5 days in the futures. In this paper the data was transformed by three days moving average therefore, the model performance for $t+2$ is very important for evaluating the real performance of the model.

TABLE 6: OUT-OF-SAMPLE PERFORMANCE FOR THE BENCHMARK FOR 3 STEPS FORECAST

\begin{tabular}{|c|r|r|r|}
\hline & $\mathbf{t}+\mathbf{1}$ & $\mathbf{t}+\mathbf{2}$ & \multicolumn{1}{c|}{$\mathbf{t + 3}$} \\
\hline Hit Rate & 79.46 & 67.15 & 52.89 \\
\hline RMSE & 0.0066 & 0.0087 & 0.0105 \\
\hline
\end{tabular}

TABLE 7: OUT-OF-SAMPLE PERFORMANCE FOR THEINPUTS GROUP A FOR 3 STEPS FORECAST

\begin{tabular}{|l|l|r|r|r|}
\multicolumn{5}{c|}{ GROUP A FOR 3 STEPS FORECAST } \\
\hline \multirow{4}{*}{} & Futures 1 & $\mathbf{t}+\mathbf{1}$ & $\mathbf{t}+\mathbf{2}$ & \multicolumn{1}{c|}{$\mathbf{t + 3}$} \\
\cline { 2 - 5 } Hit & Futures 2 & 78.11 & 67.16 & 54.37 \\
\cline { 2 - 5 } Rate & Futures 3 & 78.47 & 67.90 & 52.77 \\
\cline { 2 - 5 } & Futures 4 & 78.60 & 69.99 & 54.00 \\
\hline \multirow{3}{*}{ RMSE } & Futures 1 & 0.0066 & 0.00866 & 0.01043 \\
\cline { 2 - 5 } & Futures 2 & 0.0066 & 0.00875 & 0.0105 \\
\cline { 2 - 5 } & Futures 3 & 0.0066 & 0.0087 & 0.01053 \\
\cline { 2 - 5 } & Futures 4 & 0.0065 & 0.0086 & 0.0105 \\
\hline
\end{tabular}

TABLE 7: OUT-OF-SAMPLE PERFORMANCE FOR THEINPUTS GROUP B FOR 3 STEPS FORECAST

\begin{tabular}{|l|l|r|r|r|}
\hline \multicolumn{2}{|c|}{} & $\mathbf{t}+\mathbf{1}$ & $\mathbf{t}+\mathbf{2}$ & \multicolumn{1}{c|}{$\mathbf{t + 3}$} \\
\hline \multirow{4}{*}{$\begin{array}{l}\text { Hit } \\
\text { Rate }\end{array}$} & S\&P 500 & 78.47 & 66.54 & 50.80 \\
\cline { 2 - 5 } & USD index & 78.47 & 66.54 & 51.91 \\
\cline { 2 - 5 } & Gold & 78.11 & 65.93 & 53.14 \\
\cline { 2 - 5 } RMSE & Heating Oil & 78.60 & 69.74 & 60.64 \\
\cline { 2 - 5 } & S\&P 500 & 0.00663 & 0.00883 & 0.01063 \\
\cline { 2 - 5 } & Dollar & 0.00653 & 0.00863 & 0.010467 \\
\cline { 2 - 5 } & Gold & 0.0066 & 0.00876 & 0.0105 \\
\cline { 2 - 5 } & Heating Oil & 0.00666 & 0.00846 & 0.0101 \\
\hline
\end{tabular}

Tables 6,7 and 8 show the results of 3 steps forecast. For inputs group A, futures contracts 1,3 , and 4 have improved the performance for $t+3$. On the other hand for inputs group $\mathrm{B}$ it is evident that heating oil spot return added to the benchmark has significantly improved the forecast for time $t+3$. The out-of-sample hit rate was $60 \%$ which is by far the best hit rate obtained for $t+3$. 


\section{Conclusion}

In this paper we presented a forecasting model for crude oil spot price for the short-term. The model is based on three layer feedforward network with backpropagation algorithm. The network structure was selected after systematic rigors tests involved large number of experiments on the crude oil data. In addition, two groups of inputs were tested, crude oil futures data, and market data which include S\&P500, gold price, Dollar index and heating oil price. The results show that using futures data mainly contracts 1,2 months to maturity has outperformed all other inputs tested for one step forecast. Moreover, strong evidence was found in support of heating oil spot price to forecast crude oil spot price for multiple steps prediction.

\section{REFERENCES}

[1] NYMEX 2006, A guide to energy hedging. NYMEX, from http://www.nymex.com/media/energyhedge.pdf

[2] M. Labonte, "The effect of oil shocks on the economy: A review of the empirical evidence," Congress Research Reports CRS., Tech. Rep. RL31608, 2004.

[3] S. Moshiri and F. Foroutan, "Forecasting nonlinear crude oil futures prices," The Energy Journal, vol. 27, pp. 81-95, 2005.

[4] W. Xie, L. Yu, S. Xu and S. Wang, "A new method for crude oil price forecasting based on support vector machines," in Lecture notes in computer science, V.N. Alexanderov et al., Heidelberg: Springer, 2006, pp. 444-451

[5] J. Liu, Y. Bai and B. Li, "A new approach to forecast crude oil price based on fuzzy neural network," in FSKD '07: Proceedings of the Fourth International Conference on Fuzzy Systems and Knowledge Discovery, pp. 273-277, 2007.

[6] L. Yu, K.K. Lai, S. Wang and K. He, "Oil price forecasting with an EMD-based multiscale neural network learning paradigm," in Lecture notes in computer science, Berlin / Heidelberg: Springer, 2007, pp. 925-932.

[7] S. Wang, L. Yu and K. K. Lai, "Crude oil price forecasting with TEI@I methodology," Journal of Systems Science and Complexity, vol. 18, pp. 145-166, 2005.

[8] M. Mittermayer and G.F. Knolmayer, "Text mining systems for market response to news: A survey," Working paper no.184 University of Bern, 2005.

[9] A. Refenes Ed., Neural networks in the capital markets, New York: John Wiley \& Sons, 1995.
[10] K. Hornik, M. Stinchcombe and H. White, "Multilayer feedforwards are universal approximators," Neural Networks, vol. 2, pp. 359-366, 1989.

[11] P. Silvapulle and I. A. Mossa, "The relation between spot and future prices: Evidence from the crude oil market," The Journal of Futures Markets, vol. 19, pp. 175-193, 1999.

[12] E. Bopp Anthony and S. Scott, "Are petroleum futures prices good predictors of cash value?" The Journal of Futures Market, vol. 7, pp. 705-719, 1978.

[13] K. Chan, "A further analysis of the lead-lag relationship between the cash market and stock index futures market," The Review of Financial Studies, vol. 5, pp. 123-152, 1992.

[14] S. Abosedra and H. Baghestani, "On the predictive accuracy of crude oil futures prices," Energy Policy, vol. 32, pp. 1389-1393, 2004.

[15] A. Coppola, "Forecasting oil price movements: Exploiting the information in future market," Working paper vol. 34, CEIS Tor Vergata, 2007. http://papers.ssrn.com/paper.taf?abstract id=967408.

[16] G. Haubrich Joseph, P. Higgins and J. Miller, "Oil prices: Backward to the future?" Federal Reserve Bank of Cleveland, 2004.

[17] C. Brooks, G. Rew Alistair and S. Ritson, "A trading strategy based on the lead-lag relationship between the spot index and futures contracts for the FTSE 100," International Journal of Forecasting, vol. 17, pp. 31-44, 2001.

[18] I. Kaastra, and M. Boyd, "Designing a neural network for forecasting financial and economic time series". Neurocomputing 10, 215-236, 1996.

[19] C., N., W. Tan, "Artificial neural networks: Applications in financial distress prediction and foreign exchange trading". Wilberto Press, Gold Coast, QLD, 2001

[20] B. Vanstone, Trading in the Australian stock market using artificial neural networks. Unpublished PhD Thesis .2005.

[21] D. McNelis Paul, Neural networks in finance gaining predictive edge in the market, Massachusetts: Elsevier Acadmic Press, 2005.

[22] R. Neuneier and H. Zimmermann Georg, "How to train neural networks," in Neural networks: Tricks of the trade, Genevieve B. Orr, Klaus-Robert Müller, Berlin: Spring, 1998, pp. 273-423.

[23] R. Grothmann, "Multi-agent market modelling based on neural networks," Unpublished PhD Thesis 2005. 